\title{
PrEP for women: An update for healthcare providers
}

\section{By Danielle P. Clement, WHNP-BC, AGNP-C, AAHIVS; Kara McGee, DMS, MSPH, PA-C, AAHIVS; and Kathryn Trotter, DNP, CNM, FNP-BC, FAANP, FAAN}

Women account for almost $20 \%$ of all new diagnoses of human immunodeficiency virus (HIV) in the United States, yet their utilization of pre-exposure prophylaxis (PrEP) to prevent HIV infection is highly underutilized. Significant racial, social, and cultural factors further marginalize those who are at increased risk. Women's healthcare providers should screen for HIV risk and offer PrEP during routine clinical encounters.

KEY WORDS: HIV infection, PrEP, women, HIV prevention, sexual health, evidence-based practice

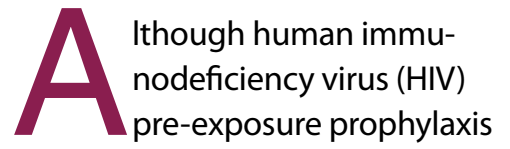

(PrEP) is a highly effective method of HIV prevention, it is largely underutilized by women, who account for almost $20 \%$ of all new HIV diagnoses in the United States. ${ }^{1,2}$ Awareness and utilization of PrEP is increasing among men who have sex with men (MSM) but continues to remain low among women, particularly among those at increased risk. The latter group includes Black women and women living in geographies with a high concentration of HIV such as the Southern region of the United States, which accounts for $52 \%$ of new HIV diagnoses. ${ }^{3}$ Trends in HIV infections further highlight the significant racial disparities that persist for Black women, whose rate of HIV acquisition is 16 times that of White women and 5 times that of Hispanic women. ${ }^{4}$ PrEP use among women has the potential to reduce new HIV infections by $61 \%$ (with $\geq 75 \%$ adherence), yet less than $10 \%$ of men and women who are "behaviorally indicated for PrEP" are utilizing this important prevention tool. ${ }^{3}$

There are many known political, cultural, and institutional barriers that increase girls' and young women's HIV risk, including poverty, gender inequality, sexual and partner 
violence, and inadequate access to educational opportunities. ${ }^{5}$ PrEP has the potential for high acceptability and uptake among women; however, access to sexual health services and knowledge of HIV prevention tools are often limited, particularly in areas where it is most needed. As barriers to access have been exacerbated by current political pressures, economic cutbacks, and health disparities intensified by the Covid-19 pandemic, connecting our most vulnerable populations with the care and prevention tools they need to live healthy lives is critical.

\section{What WHNPs/NPs need to know}

The Centers for Disease Control and Prevention (CDC) clinical guidelines for HIV PrEP are widely used to assess patients' PrEP eligibility, but they may inadequately evaluate many women who are at risk and would benefit from HIV protection. ${ }^{6}$ Because the CDC guidelines are designed to identify women "at substantial risk" for HIV acquisition, they do not fully account for a range of psychosocial and behavioral factors associated with HIV risk among women, such as education level, history of sexual violence, and HIV risk self-assessment. ${ }^{7}$ The current CDC guidelines may not consider that the person may be unaware of a partner's risks of HIV infection such as injection drug use, sexual networks, sex with male partners, recent incarceration, or HIV status. ${ }^{4}$

PrEP is largely underutilized in part because clinicians lack awareness about PrEP as an HIV prevention tool and an understanding of how to prescribe and monitor patients who are taking it. ${ }^{8}$ PrEP for HIV prevention is simple to initiate and monitor, and it has the power to substantially reduce HIV infections in women.
Nurse-led HIV prevention programs have been shown to increase uptake of PrEP. Women's health nurse practitioners (WHNPs) are in an ideal position in discussions with women concerning sexual health behavior to assess risk factors and connect those who are vulnerable to HIV infection with PrEP. ${ }^{9}$ Research has shown that individual perception of risk is generally inaccurate and often underestimated..$^{10}$ Therefore, it is critical that a patient-centered approach be used to provide accurate and objective information when counseling patients for PrEP. ${ }^{4}$

\section{Evidence in PrEP treatment}

There have been multiple, large clinical trials that have investigated the effectiveness of PrEP within populations including MSM, transgender men and women, and heterosexual men and women. Studies among heterosexual women are summarized here.

\section{Heterosexual men and women}

The Partners PrEP trial enrolled 4,758 serodiscordant heterosexual couples in Uganda and Kenya. ${ }^{11}$ Participants received either daily tenofovir disoproxil fumarate (TDF), (TDF)/ emtricitabine (FTC), or placebo for the prevention of HIV infection. The HIV-positive participants, in accordance with local treatment guidelines, were not on antiretroviral therapy. Investigators reported a $75 \%$ reduction in HIV infection among the HIV-negative partners taking TDF/FTC as well as a $67 \%$ reduction among those taking TDF alone. Among women, the estimated efficacy was $71 \%$ for TDF and $66 \%$ for TDF/FTC. The TDF2 trial in Botswana enrolled 1,219 heterosexual men and women who received daily TDF/ FTC. ${ }^{12}$ The study found a $62 \%$ reduc- tion in HIV acquisition among those taking TDF/FTC.

\section{Heterosexual women}

The FEM-PrEP study enrolled heterosexual women in South Africa, Kenya, and Tanzania to receive daily TDF/FTC or placebo to prevent HIV infection. ${ }^{13}$ The study was stopped early when an interim analysis determined that the trial was unlikely to show efficacy. The Vaginal and Oral Interventions to Control the Epidemic (VOICE) trial enrolled 5,029 heterosexual women to receive either daily oral TDF, daily oral TDF/ FTC, topical vaginal tenofovir, or corresponding placebo. ${ }^{14}$ Based on plasma tenofovir levels, adherence was approximately $30 \%$. The trial was stopped early due to futility.

\section{Women who should be considered for PrEP}

Heterosexually active women who have had sex in the past 6 months, do not have an acute or established HIV infection, and are not in a monogamous partnership with a recently tested HIV-negative partner are considered PrEP eligible if their situation or sexual behavior includes at least one of the following risks²:

- Infrequent use of condoms during sex with one or more partners of unknown HIV status.

- In an ongoing sexual relationship with an HIV-positive partner.

- Diagnosed with or reported as having a bacterial sexually transmitted infection (STI), such as gonorrhea, chlamydia, or syphilis within the past 6 months (Table 1).

\section{Regimens approved for HIV PrEP}

In July 2012, the US Food and Drug Administration (FDA) approved oral fixed-dose combination tenofovir DF-emtricitabine in a single daily dose for prevention of sexually ac- 
Table 1. Women candidates for HIV pre-exposure prophylaxis ${ }^{2}$

Without acute or established HIV infection

Any sex with opposite sex partners in past 6 months

Not in a monogamous partnership with a recently tested HIV-negative partner

And at least one of the following:

Infrequent use of condoms during sex with 1 or more partners of unknown HIV status

In an ongoing sexual relationship with an HIV-positive partner

A bacterial STI (syphilis, gonorrhea, chlamydia) diagnosis or reported in the past 6 months

HIV, human immunodeficiency virus; STI, sexually transmitted infection.

quired HIV infection among MSM, heterosexual men and women, and transgender women who have sex with men. ${ }^{2}$ TDF/FTC contains 300 $\mathrm{mg}$ of tenofovir DF and $200 \mathrm{mg}$ of emtricitabine, and is not recommended for people who have an estimated creatinine clearance of less than $60 \mathrm{~mL} / \mathrm{min}$. The most common reported side effects in several large clinical trials that used TDF/FTC were nausea and decreased appetite. ${ }^{2}$

Although TDF/FTC can cause renal dysfunction, adverse renal events in the large clinical trials of TDF/FTC for PrEP were rare. ${ }^{11}$ Outcome data from the use of TFD/FTC among pregnant women showed no evidence of adverse effects to fetuses exposed to these medications. ${ }^{12,13}$

\section{Lab evaluation and counseling at initiation of PrEP}

Baseline laboratory studies including renal function, viral hepatitis serology, STI testing, and HIV testing using an HIV-1/2 antigen-antibody immunoassay should be performed within 1 week of starting PrEP.2 Oral rapid tests are not recommended for HIV testing prior to starting PrEP (Table 2).

When starting PrEP, clinicians should educate patients on how to take their medication, common side effects, and how to handle them. The most common side effects with PrEP initiation include mild gastroin- testinal symptoms, which generally resolve within the first month of use. ${ }^{14}$ Patients should be counseled about the potential for these side effects and to continue with daily adherence. If side effects cannot be tolerated, patients should consult with their providers. In addition, clinicians should provide patients with resources such as evidence-based, reliable websites to which they can refer if questions arise. For many people, PrEP is one facet of a broader approach to HIV and STI prevention. Offering patientcentered counseling to support risk-reduction efforts is an important component of PrEP care.

\section{Time to achieve suppression}

The time from first dose of TDF/FTC to tissue concentrations that offer maximal protection against HIV infection is unknown. Pharmacokinetic studies suggest that oral TDF attains maximal concentration in rectal tissues in about 7 days, cervicovaginal tissues at about 20 days, and blood at about 20 days. ${ }^{15-17}$ It is important to counsel patients that time to protection from PrEP is not definitively known. Guidelines recommend counseling patients that optimal protection is achieved for rectal exposure after 7 days of daily dosing of TDF/FTC, and that optimal protection is achieved for all sites of exposure after 20 days of daily dosing. ${ }^{18-20}$

\section{Ongoing monitoring}

Once PrEP is established, patients should return approximately every 3 months for monitoring and lab work to ensure safety and efficacy (Table 2). There is a small risk of osteopenia with TDF use, although it is not considered clinically significant and does not require monitoring of bone mineral density. ${ }^{21} \mathrm{~A}$ risk of nephrotoxicity, particularly with longterm TDF use among people living with HIV occurs with an incidence of 1.09/1,000 person-years and is reversible on discontinuation of TDF. ${ }^{21}$ Discussions about "seasons of risk" and life circumstances such as stable partnerships, trying to conceive, and major life events should be considered and addressed at each follow-up visit to identify need for ongoing PrEP.22 Given changes to clinical practice in the era of Covid-19, creative approaches to PrEP monitoring using telemedicine and self-collected HIV testing is currently being studied and evaluated. ${ }^{23}$

\section{Pregnancy, breastfeeding, PrEP use}

The use of PrEP in pregnancy and breastfeeding is a safe and effective method for reducing HIV infection. A systematic review of PrEP use in 
Table 2. Monitoring for individuals receiving PrEP2

\begin{tabular}{l|l|l|l} 
Laboratory test & Baseline & At least every $\mathbf{3}$ months & At least every $\mathbf{6}$ months \\
\hline HIV screening test & $\mathrm{X}$ & $\mathrm{X}$ & \\
\hline Hepatitis B screening ${ }^{\star}$ & $\mathrm{X}$ & & \\
\hline Hepatitis C screening & $\mathrm{X}$ & & \\
\hline Serum creatinine with eCRCl & $\mathrm{X}$ & & $\mathrm{X}$ \\
\hline STI testing & $\mathrm{X}$ & $\mathrm{X}$ & $\mathrm{X}$ \\
\hline Pregnancy test for women & $\mathrm{X}$ & $\mathrm{X}$ &
\end{tabular}

anti-HBc, antibody hepatitis B core antigen; anti-HBs, hepatitis B surface antibody; eCrCl, estimated creatinine clearance; HBsAg, hepatitis B surface antigen; HBV, hepatitis B virus; HIV, human immunodeficiency virus; PrEP, pre-exposure prophylaxis; STI, sexually transmitted infection; TDF/FTC, tenofovir/emtricitabine.

*Includes HBsAg, anti-HBc, and anti-HBs

Note: Renal function: A serum creatinine test should be done and an eCrCL should be calculated using the Cockcroft-Gault formula. Any person with an eCrCl of $<60 \mathrm{~mL} / \mathrm{min}$ should not be prescribed TDF/FTC. Hepatitis serology: Sexually active adults are at risk for acquiring viral hepatitis B and C infection. Any person found to be HBsAg positive should be

further evaluated for management of hepatitis B. HBV infection is not a contraindication for PrEP, but persons with HBV infection require additional evaluation. Persons who are not immune to hepatitis B should be offered vaccination. Baseline screening for hepatitis C infection with a hepatitis $\mathrm{C}$ antibody test should be performed prior to starting PrEP.

women did not find any safety-related concerns to warrant prohibiting or discontinuing PrEP during pregnancy or lactation. ${ }^{24}$ Given that pregnancy is a time of particular HIV vulnerability and the clinical implications of HIV acquisition in pregnancy are significant, many experts recommend PrEP specifically during pregnancy for at-risk women. 25,26

There is a small potential for increased loss of bone mineral density (BMD) with TDF-based PrEP during pregnancy and breastfeeding. However, there is limited research in this area and loss of BMD is naturally occurring during this time. ${ }^{27}$ Pharmacokinetic studies of TDF (which is quickly absorbed and converted to tenofovir in the gut) have shown it to have poor absorption and bioavailability in breastmilk, with minimal exposure to the infant. ${ }^{27}$ The use of PrEP during pregnancy and breastfeeding has the potential to eliminate vertical transmission of HIV and improve maternal and child health outcomes globally.

\section{Access for all women}

New HIV diagnoses among women decreased from 2014 to 2018, but Black women in the United States remain at highest risk of HIV infection. ${ }^{1}$
Unfortunately, PrEP uptake among Black women is lower when compared with White women. Analysis of prescription data from 2014 to 2016 showed that among the female PrEP users for which race/ethnicity data were available, only $26 \%$ were Black (48\% were White). ${ }^{28}$ There are numerous barriers to PrEP use among Black women, including underestimation of risk by healthcare providers, lack of access to providers who prescribe PrEP, medical mistrust, and healthcare providers who have racial or other biases that negatively impact clinical decision making. ${ }^{29}$

\section{Future of PrEP}

Several novel PrEP agents are currently in various stages of clinical trials and regulatory review including intramuscular (IM) injectables, vaginal rings, implants, patches, inserts, enemas, and douches. ${ }^{30}$ Cabotegravir long-acting IM injection is currently in phase 3 clinical trials (this may change by publication) for MSM, transgender women (TGW), and cisgender women in Sub-Saharan Africa with promising results. ${ }^{30}$

In A Study to Prevent Infection with a Ring for Extended Use (ASPIRE), a monthly vaginal ring containing dapivirine has shown evidence of HIV protection in women ages 18 to 45 years living in Malawi, South Africa, Uganda, and Zimbabwe. ${ }^{31}$ Clinical studies evaluating coformulation with levonorgestrel for combined HIV protection and contraception are currently undergoing review. ${ }^{32} \mathrm{~A}$ subdermal implant (similar to the etonogestrel implant) containing the drug islatravir would provide protection for up to 1 year and is currently being investigated for safety. ${ }^{33}$

The use of "on-demand" PrEP (taken 2-24 hours before sexual encounter and 2 days after) has only been studied in MSM populations and rectal tissue (ANRS IPERGAY trial and iPREX study) where drug concentrations reach optimal levels quicker. Given the lack of research on the use of "on-demand" PrEP in women, and the potential for confusion around dosing, female patients should be counseled on the importance of daily adherence. The challenges with daily oral PrEP highlight the need for the availability of long-acting and on-demand options specifically for women that can provide consistent protection while reducing adherence barriers. 


\section{Conclusion}

PrEP is a powerful prevention tool that has the potential to significantly reduce, if not nearly eliminate, new HIV infections. There is a clear need to scale up PrEP use among women who are at risk for HIV infection, but it takes providers having the training and knowledge base to appropriately connect patients with this prevention tool. This includes a need for providers to address and confront racial biases within their own clinical practice to ensure equitable, highquality care for all patients. HIV risk assessment should become a routine part of sexual health discussion and screening process, and initiating and managing PrEP a common practice for nurse practitioners and primary care practices.

Danielle P. Clement is an adult and women's health nurse practitioner/ clinical research provider at the UNC Institute for Global Health and Infectious Diseases at the University of North Carolina-Chapel Hill. Kara McGee is Associate Clinical Professor and Kathryn Trotter is Associate Professor at the Duke University School of Nursing in Durham, North Carolina. The authors have no actual or potential conflicts of interest in relation to the contents of this article.

\section{References}

1. Centers for Disease Control and Prevention. HIV Surveillance Report: Diagnosis of HIV Infection in the United States and Dependent Areas. 2018; https://www.cdc.gov/ hiv/pdf/library/reports/surveillance/ cdc-hiv-surveillance-report-2018updated-vol-31.pdf.

2. Centers for Disease Control and Prevention. Preexposure Prophylaxis for the Prevention of HIV Infection in the United States - 2017 Update: A Clinical Practice Guideline. 2017.

3. Siegler AJ, Mouhanna F, Giler RM, et al. The prevalence of pre-ex- posure prophylaxis use and the pre-exposure prophylaxis-to-need ratio in the fourth quarter of 2017 , United States. Ann Epidemiol. 2018;28(12):841-849.

4. Aaron E, Blum C, Seidman D, et al. Optimizing delivery of HIV preexposure prophylaxis for women in the United States. AIDS Patient Care STDS. 2018;32(1):16-23.

5. McGovern T, Fine J, Crisp C, Battistini E. As the HIV epidemic among young women grows, can we look to the SDGs to reverse the trend? Health Hum Rights. 2017;19(2):223236.

6. Calabrese SK, Willie TC, Galvao RW, et al. Current US guidelines for prescribing HIV pre-exposure prophylaxis (PrEP) disqualify many women who are at risk and motivated to use PrEP. J Acquir Immune Defic Syndr. 2019;81(4):395-405.

7. Patel AS, Goparaju L, Sales JM, et al. Brief report: PrEP eligibility among at-risk women in the southern United States: associated factors, awareness, and acceptability. J Acquir Immune Defic Syndr. 2019;80(5):527-532.

8. Smith DK, Mendoza MC, Stryker JE, Rose CE. PrEP awareness and attitudes in a national survey of primary care clinicians in the United States, 2009-2015. PLoS One. 2016;11(6):e0156592.

9. Selfridge M, Card KG, Lundgren $\mathrm{K}$, et al. Exploring nurse-led HIV pre-exposure prophylaxis in a community health care clinic. Public Health Nurs. 2020;37(6):871-879.

10. Sales JM, Sheth AN. Associations among perceived HIV risk, behavioral risk and interest in PrEP among Black women in the southern US. AIDS Behav. 2019;23(7):1871-1876.

11. Baeten JM, Donnell D, Ndase P, et al. Antiretroviral prophylaxis for HIV prevention in heterosexual men and women. $N$ Engl J Med. 2012;367(5):399-410.

12. Thigpen MC, Kebaabetswe PM, Paxton LA, et al. Antiretroviral preexposure prophylaxis for heterosexual HIV transmission in Botswana. N Engl J Med. 2012;367(5):423-434.
13. Van Damme L, Corneli A, Ahmed K, et al. Preexposure prophylaxis for HIV infection among African women. $N$ Engl J Med. 2012;367(5):411-422.

14. Marrazzo JM, Ramjee G, Richardson BA, et al. Tenofovir-based preexposure prophylaxis for HIV infection among African women. N Engl J Med. 2015;372(6):509-518.

15. Mugwanya KK, Wyatt C, Celum C, et al. Changes in glomerular kidney function among HIV-1-uninfected men and women receiving emtricitabine-tenofovir disoproxil fumarate preexposure prophylaxis: a randomized clinical trial. JAMA Intern Med. 2015;175(2):246-254.

16. Antiretroviral Pregnancy Registry Steering Committee. Antiretroviral Pregnancy Registry Interim Report for 1 January 1989 through 31 January 2020. Wilmington, NC: Registry Coordinating Center; 2020.

17. Wang L, Kourtis AP, Ellington S, et al. Safety of tenofovir during pregnancy for the mother and fetus: a systematic review. Clin Infect Dis 2013;57(12):1773-1781.

18. Anderson PL, Kiser JJ, Gardner EM, et al. Pharmacological considerations for tenofovir and emtricitabine to prevent HIV infection. J Antimicrob Chemother 2011;66(2):240-250.

19. Patterson KB, Prince HA, Kraft E, et al. Penetration of tenofovir and emtricitabine in mucosal tissues: implications for prevention of HIV-1 transmission. Sci Transl Med. 2011;3(112):112re114.

20. New York State Department of Health AIDS Institute Clinical Guidelines Program. PrEP to Prevent HIV and Promote Sexual Health. 2020.

21. Riddell Jt, Amico KR, Mayer KH. HIV preexposure prophylaxis: a review. JAMA. 2018;319(12):1261-1268.

22. Seifert SM, Glidden DV, Meditz AL, et al. Dose response for starting and stopping HIV preexposure prophylaxis for men who have sex with men. Clin Infect Dis. 2015;60(5):804-810.

23. Hoagland B, Torres TS, Bezerra DRB, et al. Telemedicine as a tool for PrEP delivery during the 
COVID-19 pandemic in a large HIV prevention service in Rio de Janeiro-Brazil. Braz J Infect Dis. 2020;24(4):360-364.

24. Davies N, Heffron R. Global and national guidance for the use of pre-exposure prophylaxis during peri-conception, pregnancy and breastfeeding. Sex Health. 2018;15(6):501-512.

25. Zorrilla C, Báez F, Colón K, et al. HIV seroconversion during pregnancy and the need for pre-exposure prophylaxis (PrEP). HIV AIDS (Auckl). 2018;10:57-61.

26. Joseph Davey DL, Pintye J, Baeten $\mathrm{JM}$, et al. Emerging evidence from a systematic review of safety of pre-exposure prophylaxis for pregnant and postpartum women: where are we now and where are we heading? J Int AIDS Soc. 2020;23(1):e25426.

27. Hu X, Wang L, Xu F. Guides concerning tenofovir exposure via breastfeeding: a comparison of drug dosages by developmental stage. Int J Infect Dis. 2019;87:8-12.
28. Huang Y-LA, Zhu W, Smith DK, et al. HIV preexposure prophylaxis, by race and ethnicity - United States, 2014-2016. MMWR Morb Mortal Wkly Rep. 2018;67(41):11471150 .

29. Ojikutu BO, Amutah-Onukagha $\mathrm{N}$, Mahoney TF, et al. HIV-related mistrust (or HIV conspiracy theories) and willingness to use PrEP among Black women in the United States. AIDS Behav. 2020;24(10):2927-2934.

30. Clement ME, Kofron R, Landovitz RJ. Long-acting injectable cabotegravir for the prevention of HIV infection. Curr Opin HIV AIDS. 2020;15(1):19-26.

31. Baeten JM, Palanee-Phillips T, Brown ER, et al. Use of a vaginal ring containing dapivirine for HIV-1 prevention in women. $N$ Engl J Med. 2016;375(22):21212132.

32. Dallal Bashi YH, McCoy CF, Murphy DJ, et al. Towards a dapivirine and levonorgestrel multipurpose vaginal ring: investi- gations into the reaction between levonorgestrel and addition-cure silicone elastomers. Int J Pharm. 2019;569:118574.

33. Cambou MC, Landovitz RJ. Novel antiretroviral agents. Curr HIV/ AIDS Rep. 2020;17(2):118-124.

\section{CALL FOR MANUSCRIPTS Focus on High-Risk Pregnancy and Complications in Pregnancy}

Women's Healthcare: A Clinical Journal for NPs, the official, peer-reviewed journal of the National Association of Nurse Practitioners in Women's Health, invites you to submit manuscripts with a focus on assessment and management for high-risk pregnancy conditions and/or prevention, assessment, and management of complications in pregnancy. Other obstetric topics of interest include considerations and innovations in providing prenatal/postpartum care for specific populations (eg, adolescents, homeless individuals, individuals with disabilities, transgender individuals) and addressing inequities in obstetric care.

We are interested in manuscripts for feature-length articles or short-form articles to include Assessment and management, Clinical resources, and On the case. Consult our Guidelines for authors for more detailed information.

Email queries concerning manuscript submission to gschwenker@ healthcommedia.com. 